\title{
Fluoride Content in Bottled Water in Fiji
}

\author{
Anumala Ram, Seema Lal* \\ Department of Oral Health, College of Medicine, Nursing and Health Sciences, Fiji National University, Brown Street, Suva, Fiji
}

\begin{abstract}
Objectives: The aims of this study was to evaluate the fluoride content of bottled drinking water co mmercially available in Fiji in March 2006; and to report on the labelling of fluoride concentrations. Methods and Materials: Commercially available bottled water in Fiji, in March 2006 was purchased from supermarkets. Three bottles of 11 brands were purchased. Ten millilitres of each water sample was mixed with 2 millilitres of spands reagent; these were then tested for fluoride concentrations using photo spectrometry at wavelength of 580 nano metres. The fluoride concentrations listed on the sample labels were also noted and compared with the results obtained from the spectrometry test. Results: The mean ( \pm standard deviation) of fluoride was $0.09( \pm 0.05) \mathrm{ppm}$. None of the brands tested labelled the fluoride concentration in the bottled water. The range of fluoride concentration was between 0.02 to $0.34 \mathrm{ppm}$. There was also a vast variation of fluoride concentrations within the same brand of product. Conclusions: Co mmercia lly available bottled drin king waters in Fiji contain a wide range of concentrations of fluoride and none of the manufacturers labelled the content of fluoride on the bottle. It was also noted that none of the brands contained optimal levels of fluoride.
\end{abstract}

Keywords Bottled Water, Fluoride, Concentration, Labelling

\section{Introduction and Objectives}

There has been increased efforts and awareness fluoridating the community drinking water supply in Fiji. The patterns of water consumption in terms of source of drinking water, amounts and frequency has become important to have optimum caries preventive effect of fluoridated water. Establishing other sources of fluoride is important in order to determine the ideal level of concentration of fluoride in the community water supply. There has been an evolving trend in consumption of bottled water in Fiji recently. With a vast range of brands becoming available in the market with various prices range and differences in content and higher utilization among consumers.

Setting ideal levels of fluoride in community water supply, determining additional sources of fluoride is more important in children under the age of 6 years to prevent the risk of developing fluoros is in both permanent and primary teeth. Fluorosis occurs when the optimum levels of fluoride exceeds $1 \mathrm{ppm}$ and disturbs the enamel minerlisation stage and results in a range of defects affecting the amount and quality of enamel. The severity depends on the amount of fluoride exposure and stage of tooth development.

Correct labelling of contents of foods and beverages is one of the ways of ensuring that consumers are making informed decisions. The concentration of some elements

\footnotetext{
* Corresponding author:

seema.lal@fnu.ac. fj (Seema Lal)

Published online at http://journal.sapub.org/phr

Copyright (C) 2012 Scientific \& Academic Publishing. All Rights Reserved
}

such as calcium, sodium and aluminium in bottled water is regulated in some countries[1], however, there is no regulation regarding fluoride content of bottled water in Fiji.

Manufacturers are encouraged to list the nutritional contents of their products, but labelling of fluoride levels on bottled water is not legally required. When fluoride concentrations appear on the bottle labels, they may not always be accurate. Weinberger found the accuracy of the printed concentrations of fluoride to be doubtful in 16 of 17 bottles tested in a Canadian study[2]. Another study done in the United Kingdom showed that only 6 of 26 bottles tested had the fluoride concentrations printed on their labels[3]. Similar study done in Australia showed that the amount of fluoride in still bottled water was neglible with all having a fluoride content below $0.08 \mathrm{ppm}$ and only 3 out of 10 brands indicated fluoride content on their label [4].

A study done in Mexico showed that the fluoride concentrations in bottled water was lower than in other drinks such as juices, nectars and carbonated drinks. In another study done in Toluca City, Mexico the mean fluoride concentrations in bottled water was $0.34 \mathrm{ppm}$ (range $=0.052-0.48 \mathrm{ppm}$ ), which was not in the recommended optimal range[5].

A study done in Greece showed that most of the bottled water tested contained less than $0.3 \mathrm{ppm}$ fluoride, but was as high as $4.8 \mathrm{ppm}$ in one of the brands of bottled water, and most brands did not mark fluoride content on their labels.

It is important to know precisely the concentration of fluoride in the water that a patient is drinking. These data should be of use to dentists both in clinical practice and 
dental public health, providing them with current information necessary when advising on fluoride supplementation both on an individual and community basis to propose an effective preventive program with a minimum risk of fluorosis[6]. This information is also vital to consumers to assist them in making informed decisions.

The trends of water and beverage consumption are changing; many people in Fiji are opting for bottled water today and their use is increasing by the day. Some commercially available bottled water does not contain fluoride, while others do. There has not been any study done in Fiji that has evaluated the fluoride content of bottled water. People pay more for bottled water; they should be able to get good value for their money and all the possible benefits that they can get.

This study will identify the commercially available bottled water in Fiji that contain fluoride and those that do not contain fluoride; and if the fluoride that they contain are of optimal levels. The findings of this study will help the public to make an informed decision.

\section{Methods and Materials}

An experimental study was carried out where the fluoride content of commercially available bottled water was measured. The results obtained were compared to the recommended optimal fluoride levels in water. The differences in fluoride concentration between and within brands were also compared; and the content of fluoride displayed on the label of the samples was noted.

Bottles of 11 commercially available brands of water were obtained from supermarkets around Suva, Fiji in March 2006, of these, $55 \%$ (6 brands) had their source or production in Fiji. Three bottles of each brand were purchased. The labels of the bottles were studied to check for labelling of fluoride concentrations. All bottles were stored in a dark place and in their original closed plastic containers at room temperatures until the fluoride analysis was done.
The samples were analyzed at a Water Treatment Laboratory, in Nasinu, Fiji. Spectrophotometry was used to analyze the fluoride concentration in the samples. A spectrometer is a device consisting of a light source, some optics for focusing light, prism or diffraction grating, a sample compartment to hold a transparent tube, a light sensitive detector (this converts light intensity into an electric current) and electronics for measuring and displaying the output of the detector.

This method involved measuring the intensity of a colour in a sample solution and relating it to the concentration of the analyte; the concentration of the sample appeared on the automatic output detector of the device. When light is passed from the pris $m$ through a narrow opening to the sample, the pris $m$ rotates to select the colour (wavelength) of light and to match the colour that the sample has absorbed. Light from the sample then reaches the detector, and the detector converts the intensity of colour into concentration of fluoride.

The control solution ( 10 millilitres of $d$ istilled water) was tested for fluoride concentration first. Then, the standard solutions of known fluoride concentrations were tested to check for the reliability of the technique.

After shaking the bottle of water, a 10-millilitre sample was pipetted and mixed with 2 millilitres of Spands reagent. This reagent helps in detection of the colour that shows the wavelength of fluoride; which is detected at 580 nanometres.

All the data was entered and analyzed using SPSS (Statistical Package for Social Sciences) and Microsoft Excel.

\section{Results}

The table below shows the 11 brands of bottled water tested. Regarding the quality of the labelling of bottled waters, none of the samples had fluoride content listed on the labels. 7 out of 11 brands (63\%) tested had their source or production site in Fiji.

Table 1. Fluoride Concentrations of 11 Bottled Water (mean +/- SD)

\begin{tabular}{cccc}
\hline Bottled Water Brand & Country of Origin & Fluoride Concentration (ppm) & Fluoride Labelling on Bottle \\
\hline Aqua Pacific & Fiji & $0.07 \pm 0.04$ & Not Labelled \\
Aqua Safe & Fiji & $0.05 \pm 0.00$ & Not Labelled \\
Bleu & Malaysia & $0.14 \pm 0.18$ & Not Labelled \\
Bula Purified Water & Fiji & $0.05 \pm 0.03$ & Not Labelled \\
Diamond Aqua Fiji & Fiji & $0.06 \pm 0.01$ & Not Labelled \\
Fiji Natural Artesian Water & Fiji & $0.20 \pm 0.11$ & Not Labelled \\
H2GO Pure & New Zealand & $0.06 \pm 0.01$ & Not Labelled \\
Hawaiian Springs & Fiji & $0.05 \pm 0.03$ & Not Labelled \\
Island Chill & Fiji & $0.14 \pm 0.12$ & Not Labelled \\
Black And Gold Natural Spring Water & Australia & $0.05 \pm 0.00$ & Not Labelled \\
Pump Pure Spring Water & New Zealand & $0.10 \pm 0.04$ & Not Labelled \\
\hline
\end{tabular}




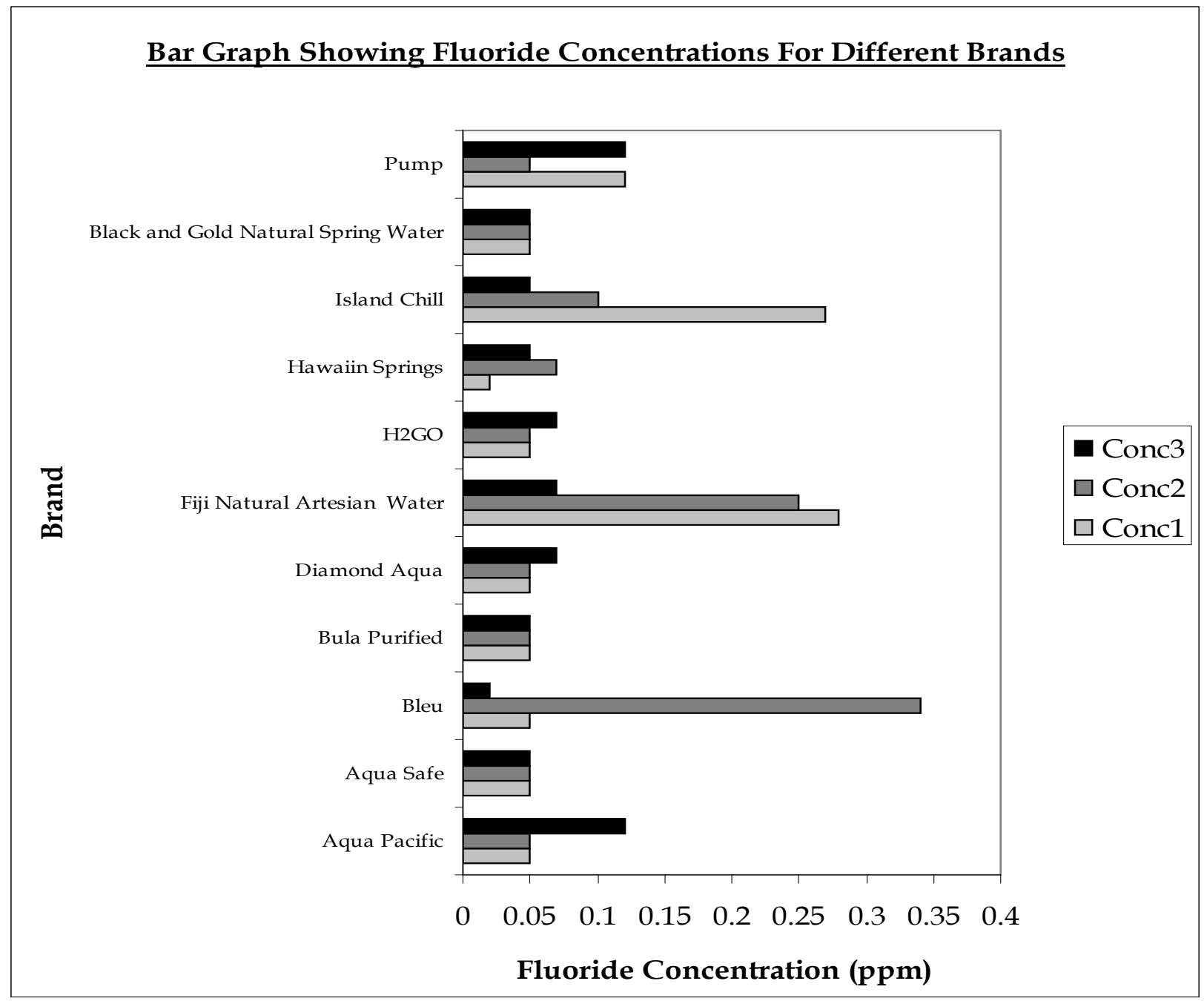

Figure 1. Fluoride Concentration for Different Brands of Bottled Water

It was found that none of the sample brands contained the optimum levels of fluoride and all the samples had fluoride concentrations below the optimum level; that is required for preventing dental caries without causing dental fluorosis. The most common fluoride concentration that observed was $0.05 \mathrm{ppm}(54.55 \%)$ with a mean $(+)$ standard deviation fluoride concentration of bottled water samples was $0.09+0.05 \mathrm{ppm}$.

Out of the eleven brands tested; 3 brands (27.3\%); Black and Gold Natural Spring Water, Aqua Safe, and Bula Purified Water had consistent readings of the fluoride concentration within the samples tested. The other eight brands tested had variations in the fluoride concentration within the sample and the concentrations ranged from 0.02 - $0.34 \mathrm{ppm}$. Figure 1 shows concentrations of fluoride within and between samples tested for fluoride content in eleven commercially available bottled water

\section{Discussion}

The concentration of fluoride in bottled drinking water purchased from local stores was found to vary widely, which ranged between 0.02 to $0.34 \mathrm{ppm}$ with a mean concentration of $0.09 \pm 0.05 \mathrm{ppm}$. This variation was consistent with similar studies done in other countries $[1,2,6,8,9]$. None of the tested samples displayed the fluoride content of the water on the labels. This study showed that for one of the tested brands of bottled water, $\mathrm{Bleu}$, the fluoride concentration was $0.34 \mathrm{ppm}$. This extremely high fluoride concentration can cause fluorosis if young children consume the water alone or in addition to other fluoride supplements as home care use.

The overall public water supply in Fiji is non-fluoridated and the only sources of fluoride that majority of the people have access to; are over-the-counter products and professionally applied fluorides. Both the mentioned sources are expensive and limits their accessibility to majority of the population; there are cheaper non-fluoridated toothpastes available contributing to the fluoride barrier until 2011 when it was officially removed from the market. The exposure to fluoride is further compromised due to socio-economic and political factors; however, consumers can choose to obtain potential 
preventive benefits from bottled water if it contained optimal levels of fluoride.

The results obtained in this study are similar to research done in Greece $(0.35 \pm 1.00 \mathrm{ppm})$. The Greek[6] study showed that the mean fluoride concentration of commercially available water was higher in comparison to the results obtained in this study $(0.09 \pm 0.05)$. There was also a difference in the range of fluoride concentrations in the both studies; the range of fluoride ( 0.02 to $0.34 \mathrm{ppm})$ for the present study was relatively lower than the Greek study ( 0.05 to $4.8 \mathrm{ppm})$. The range of values obtained in the Greek study was wider with values of some brands exceeding the recommended mean concentration of fluoride in drinking water. In the Greek study $18 \%$ of the brands labelled the content of fluoride; whereas none of the brands of bottled water tested in this study contained fluoride content labels.

Findings from another study done in Mexico[5] showed higher value for mean concentration of fluoride in bottled water $(0.21 \pm 0.08 \mathrm{ppm})$ in comparison to the present study $(0.09 \pm 0.05) \mathrm{ppm}$.

The values for the mean fluoride concentration obtained in the present study $(0.09 \pm 0.05 \mathrm{ppm})$ were similar to that of a study done in the United Kingdom $(0.08 \pm 0.08$ $\mathrm{ppm}$ [1]. The values obtained for the range in the two studies were also comparable, with the United Kingdom study having a range of 0.01 to $0.37 \mathrm{ppm}$ and the present study with a range of 0.02 to $0.34 \mathrm{ppm}$. W ith regards to the quality of labelling in the United Kingdom study, $32 \%$ of the brands had the content of fluoride labelled which is higher in comparison to quality of labelling of Greek study $(18 \%)$ and the present study where none of brands labelled the content of fluoride on their bottled water. Furthermore, the United Kingdom study also showed that the content of fluoride displayed on the labels varied from the values obtained through the tests.

There was considerable variation in the content of fluoride within the brands of bottled water tested in the present study. This finding is consistent with the findings from the Mexico study where there were large variations in fluoride concentration within and between the brands tested. In contrast, to the United Kingdom study, there were no considerable differences between three batch numbers for each brand, which were analy zed.

The dental health of children who utilize bottled water as the primary source of drinking water may be significantly affected in one the ways: they may receive an appropriate level of fluoride from bottled water, allowing for optimal caries prevention, or receive suboptimal levels of fluoride, with a resultant increase in dental caries. Finally, an elevated level of fluoride in child ren's drinking water could result in fluorosis. In Fiji there is no official regulation defining the limits of fluoride in bottled water, in other countries the highest limit has been set at 4 ppm [10].

It is important that manufacturers recognize and monitor fluoride concentrations, especially in water they are using to manufacture their products. If necessary, fluoride levels should be modified in the final product. Dentists should be aware of the fluoride concentrations of the drinking water of their child patients when prescribing fluoride supplements.

The wide ranges of fluoride concentrations in bottled water make the real fluoride intake by the populations very difficult to assess. Wide variations in dietary patterns add further to the complexity.

All fluoride sources are important, and the total fluoride intake from all sources is critical in the development of fluorosis $[11,12,13,14,15,16]$. The amount of fluoride necessary to cause fluorosis is not precisely known, and variations among individuals exist [11]. In infancy the ma in fluoride sources are thought to be from commercially available beverages and foods used during weaning, a period that is coincides with the stage of calcification in several developing permanent tooth crowns [17]. In addition to fluoride concentrations in products, the quantities consumed and the age of the child are also important [11].

The level of fluoride in bottled drinking water has implications for the prescription of fluoride supplements as well as for the estimation of the risk of fluorosis. The first step towards evaluating how bottled water consumption might affect fluoride exposure is to determine the fluoride content of bottled water. It is equally important to evaluate daily water intake among children living in different areas, with respect to their age, gender, and seasonal changes of temperature. Based on this information, the total fluoride intake from different sources, and consequently the risk for fluorosis can be estimated [6].

It has been suggested that if the level of fluoride in drinking water is below $0.7 \mathrm{ppm}$, supplements maybe required in some cases, whereas if the level is greater than $0.7 \mathrm{ppm}$, supplementation is not necessary $[18,19]$. Furthermore, if a high concentration of fluoride in drinking water is used to make up baby formula that have been shown to contain variable amounts of fluoride themselves, then potentially very high intakes of fluoride are possible in young children [20,21].

Some of the shortcomings of the present study are that only three samples of each brand were tested; however, this coincides with the United Kingdom and the Greek study that also used three samples per brand. In contrast, the study done in Mexico used only one sample per brand. The study used only one of the two available methods for testing fluoride since it was the most accessible one. The study has important implications since it is the first study done in Fiji that measured the fluoride concentration of bottled water and all the commercially available bottled water was tested.

In view of the wide variation of fluoride concentration in the tested bottled waters, regulatory guidelines for controlling concentration in order to provide caries preventive effects and prevent dental fluorosis are 
recommended. There is an emerg ing trend in Fiji where more people are resorting to bottled water for convenience and safety. It is recommended that further research is done to determine the number of people consuming bottled water in Fiji and extent of usage among children.

\section{Conclusions}

This study has shown that commercially available bottled drinking waters in Fiji contain a wide range of concentrations of fluoride and none of the manufacturers labelled the content of fluoride on the bottle. Labelling the content of fluoride is vital consumer information especially in paediatric patients since this allows parents to make informed decisions.

It was also noted that none of the brands contained optimal levels of fluoride that is needed to prevent dental caries without causing dental fluorosis. In patients who receive fluoride from other sources and at the same time have a high consumption of bottled water maybe at risk of developing dental fluorosis. However, patients who totally rely on bottled water as their source of fluoride may not be receiving optimum levels to have a caries preventive effect.

When prescribing fluoride supplements, dentists should be aware of the fluoride content of bottled waters used by paediatric patients, especially brands with a concentration higher than $0.3 \mathrm{ppm}$.

Fiji is planning to fluoridate its water supply, this raises the concern that if bottled water is regularly consumed an effective means of preventing dental caries will be unavailable if bottled water is the only source of drinking water.

The limitations of this study included not making available a sample of reticulated water for analysing fluoride content for comparison with samples from bottled water. Secondly, the method used to analyse fluoride in this study was photo spectrometry due to lack of resources and availability of laboratory with such facilities. Other methods such as an ion analyser or fluoride selective electrode, allow regulation of ion strength of a solution and control concentration of hydroxide ions and interfering ions of metals. Other limitations include storage of the sample and preparation of the sample in order to adjust the ionic strength and $\mathrm{pH}$ of the sample.

There needs to be public awareness of the potential risk of losing the benefits of caries prevention by drinking water from a source which is optimally fluoridated in comparis on to drinking bottled water which may not be optimally fluoridated. This has to be addressed by the health sector in Fiji to maximize the cost/benefit effects of water fluoridation and having caries prevention.

\section{REFERENCE}

[1] Fomon S J, Ekstrand J, Ekhard E, Ziegler E. Fluoride intake and prevalence of dental fluorosis: trends in fluoride intake with special attention to infants. J Pub Health Dent 2000; 60: 131-139.

[2] Zohouri F. V., Maguire A., Moynihan P. J., Fluoride Content of Still Bottled Waters Available in the North -East of England, UK, British Dental Journal, 2003, vol. 195, pp. 515-518.

[3] Wienberger S. J., Bottled Drinking Waters: are the Fluoride Concentrations Shown on the Bottles Accurate?, International Journal of Paed iatric Dentistry, 1991, vol. 1, pp. $143-146$.

[4] Cochrane NJ, Saranathan S, Morgan MV, Dashper SG, Fluoride Content of Still Bottled Water in Australia, Australian Dental Journal, 2006, 51 (3) 242-244.

[5] MacFadyen E. E., McNee S. G., Weetman D. A., Fluoride Content of Some Bottled Water Spring Waters, British Dental Journal, 1982, vol. 153, pp 423 - 424.

[6] Jimenez - Farfan M. D., Hernandez - Guerrero J. C. et.al., Fluoride Content In Bottled Waters, Juices, and Carbonated Soft Drinks in Mexico City, Mexico, International Journal of Paediatric Dentistry, 2004, vol. 14, pp. 260-266.

[7] Ahiropoulos V., Fluoride Content of Bottled Waters Available in Northern Greece, International Journal of Paediatric Dentistry, 2006, vol. 16, pp. 111-116.

[8] Russell H. H., Jackson R. J., Spath D. P., Book S. A., Chemical Contamination of California Drinking Water, West Journal of Medicine, 1987, vol. 147, pp. 615 - 622.

[9] Toumba K. J., Levy S., Curzon M. E. J., the Fluoride Content of Bottled Drinking Waters, British Dental Journal, $1994 ; 176$, pp. $266-268$.

[10] Lalumandier J. A., Ayers L. W., Fluoride and Bacterial Content of Bottled Vs. Tap Water, Archives of Medicine, 2000, vol. 9, pp. $246-250$.

[11] Kiristy M. C., Levy S. M., Warren J. J., Guha-Chowdhury N., Heilman J. P., Marshall T., Sources of Fluoride Intake in Children, Journal of the American Dental Association, 1996, vol. $127,895-902$.

[12] Fejerskov O., Thylstrup A., Larsen M. J., Clinical and Structural Features and Possible Pathogenic Mechanisms of Dental Fluorosis, Scandinavian Journal of Dental Research, 1977 , vol. $85,510-534$.

[13] Richards A., Kragstrup J., Josephsen J., Fejerskov O., Dental Fluorosis Developed in Post-Secretory Enamel, Journal of Dental Research, 1986; vol. 65, 1406-1409.

[14] Levy S. M., Maurice T. J., Jakobsen J. R., Feeding Patterns, Water Sources and Fluoride Exposures of Infants and 1-Year-Olds, Journal of the American Dental Association, 1993; vol. 124, 65-69.

[15] Clark D. C., Hann H. J., Williamson M. F., Berkowitz J., Influence of Exposure to Various Fluoride Technologies on the Prevalence of Dental Fluorosis, Community Dentistry and Oral Epidemiology, 1994; vol. 22, 461-464.

[16] Clark D.C., Trends in prevalence of dental fluorosis in North America, Community Dentistry and Oral Epidemiology, 1994; vol. 22, 148-152. 
[17] Levy S. M., Kohout F. J., Guha-Chowdhury N., Kiristy M. C., Heilman J. R., Wefel J. S., Infants' Fluoride Intake From Drinking Water Alone, Journal of Dental Research, 1995, vol. 74, 1399 - 1407.

[18] Flaitz C. M., Hill E. M., Hicks M. J., A Survey of Bottled Water Usage by Paediatric Dental Patients: Implications for Dental Health, Quintessence International, 1989, vol. 20, pp. $847-852$.

[19] Chan J. T., Stark C., Jeske A. H., Fluoride Content of
Bottled Waters: Implications For Dietary Fluoride Supplementation, Texas Dental Journal, 1990, vol. 107, pp. $17-21$.

[20] Tinanoff N., Mueller B., Fluoride Content in Milk and Formula for Infants, Journal of Dentistry for Children, 1978, vol. 45 , pp. $53-55$.

[21] Vlachou A., Drummond B. K., Curzon M. E. J., Fluoride Concentration if Infant Foods and Drinks in the United Kingdom, Caries Research, 1992, vol. 26, pp. 29-32. 